\title{
Vorwort der Gastherausgeberin
}

\author{
Susanne Feiel \\ Head of Departments, Resources Innovation Center Leoben, Leoben, Österreich
}

Angenommen 26. August 2021; online publiziert 14. September 2021

Das Resources Innovation Center - RIC Leoben bündelt internationale Ressourceninnovations- und Nachhaltigkeitsaktivitäten der Montanuniversität Leoben entlang des Lebenszyklus von Materialien und wirkt als interdisziplinärer Koordinator und Katalysator zur Themen- und Projektentwicklung mit internen, wie auch externen Stakeholdern. Dabei kann auf ein extensives europäisches Netzwerk zurückgegriffen werden, dass mittlerweile über 2000 individuelle Partner umfasst.

Seit nunmehr sieben Jahren gibt es das RIC Leoben, und es hat sich von einer kleinen Gruppe motivierter Menschen, die auf europäischer Ebene etwas verändern wollten, zu einem professionellen Team mit einer beachtlichen Erfolgsbilanz entwickelt. Nicht nur können wir auf zahlreiche erfolgreiche Projekte und Projektkoordinationen für die Universität zurückblicken, sondern auch darauf, dass wir uns zu einem Raum für zukunftsorientierten Dialog entwickelt haben, in dem die Zukunft nicht nur auf der traditionellen technologischen Ebene, wie wir sie als Universität kennen, gedacht wird, sondern eingebettet in den Kontext der Gesellschaft und ihren zahlreichen transversalen Dimensionen gesehen und neu erfunden wird. Das RIC Leoben unterstützt die Universität als neutrale Einheit, um Chancen innerhalb dieses Narrativs zu generieren, zu ergreifen und zu entwickeln, und wir freuen uns, so gemeinsam in eine neue bessere Zukunft zu gehen, die wir aktiv mitgestalten können.
Im Folgenden findet sich ein grober Überblick über die Aktivitäten des RIC Leoben der letzten sieben Jahre, der ein bisschen Inspiration für die Zukunft sein soll.

Susanne Feiel

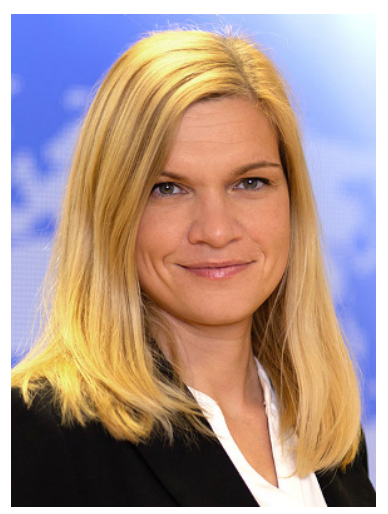

Susanne Feiel

Hüttenmännische Monatshefte 